\title{
Probe method and a Carleman function
}

\author{
Masaru IKEHATA \\ Department of Mathematics, Graduate School of Engineering \\ Gunma University, Kiryu 376-8515, JAPAN
}

20 August 2007 Final

\begin{abstract}
A Carleman function is a special fundamental solution with a large parameter for the Laplace operator and gives a formula to calculate the value of the solution of the Cauchy problem in a domain for the Laplace equation. The probe method applied to an inverse boundary value problem for the Laplace equation in a bounded domain is based on the existence of a special sequence of harmonic functions which is called a needle sequence. The needle sequence blows up on a special curve which connects a given point inside the domain with a point on the boundary of the domain and is convergent locally outside the curve. The sequence yields a reconstruction formula of unknown discontinuity, such as cavity, inclusion in a given medium from the Dirichlet-to-Neumann map. In this paper, an explicit needle sequence in three dimensions is given in a closed form. It is an application of a Carleman function introduced by Yarmukhamedov. Furthermore, an explicit needle sequence in the probe method applied to the reduction of inverse obstacle scattering problems with an arbitrary fixed wave number to inverse boundary value problems for the Helmholtz equation is also given.

AMS: 35R30, 35R25, 35J05, 33E12, 35C05

KEY WORDS: inverse boundary value problem, Laplace equation, probe method, Carleman function, Mittag-Leffler's function, harmonic function, Vekua transform, Helmholtz equation, inverse obstacle scattering problem, reconstruction formula, inclusion, cavity, crack, obstacle, electrical impedance tomography
\end{abstract}

\section{Introduction}

In [27] Isakov considered the uniqueness issue of the problem for recovering the unknown inclusions in a given electric conductive medium from infinitely many boundary data. This is a special, however, very important version of the Calderón problem [8] and is formulated as an inverse boundary value problem for an elliptic partial differential equation in which the boundary data are given by the associated Dirichlet-to-Neumann map. Therein he established a uniqueness theorem. The points of his paper are: a contradiction argument; orthogonality identities deduced by denying the conclusion, singular solutions, unique continuation theorem, Runge approximation property and uniqueness of the solution of the Cauchy problem for elliptic equations. See [15] for these points. 
After his work for almost 10 years nobody mentioned the reconstruction formula of unknown inclusions. However, finding such a formula is a natural attempt in studying inverse problems. Nowadays we can cite at least three types formulae for the full reconstruction of unknown inclusions:

- The probe method [13] in two and three dimensions.

- An application by Brühl [7] of the factorization method of Kirsch [28] in two and three dimensions.

- A solution of the Calderón problem in two dimensions by Astala-Päivärinta [3, 4].

This paper is concerned with the foundation of the probe method. The method applied to inclusions in a medium with constant conductivity is based on the existence of a special sequence of harmonic functions which is called a needle sequence and plays a role of a probe needle. The sequence blows up on a special curve which is called a needle and connects a given point inside the domain with a point on the boundary of the domain and is convergent locally outside the curve. The existence has been ensured by using the Runge approximation property which can be proved by showing the denseness of the ranges of infinitely many linear integral operators. Therefore, the construction of the needle sequence is not explicit and this makes us difficult to understand theoretically the point wise behaviour of the needle sequence on the needle.

In this paper, we give an explicit and concise needle sequence in three dimensions for a special needle given by a line segment. The idea for the construction came from reconsidering the role of a Carleman function which is a special fundamental solution with a large parameter for the Laplace operator and gives a formula to calculate the value of the solution of the Cauchy problem in a domain for the Laplace equation. Roughly speaking, we say that a function $\Phi(y, x, \tau)$ depending on a large parameter $\tau>0$ is called a Carleman function for a domain $\Omega$ and the portion $\Gamma$ of $\partial \Omega$ if it satisfies the equation $\triangle u+\delta(y-x)=0$ in $\Omega$; for each fixed $x \in \Omega \Phi(\cdot, x, \tau)$ and $\partial / \partial \nu \Phi(\cdot, x, \tau)$ on $\partial \Omega \backslash \Gamma$ vanish as $\tau \longrightarrow \infty$. This function gives a representation formula of any solution of the Cauchy problem for the Laplace equation in $\Omega$ by using only the Cauchy data on $\Gamma$ and yields a natural regularization for the numerical computation of the solution from a noisy inaccurate Cauchy data on $\Gamma$. The Cauchy problem for the Laplace equation is a fundamental and important ill-posed problem appearing in mathematical sciences, engineering and medicine. Therefore, it is quite important to seek an explicit Carleman function in several domains. For this problem Yarmukhamedov [36] gave a very interesting Carleman function for a special domain in three dimensions which is a special version of his fundamental solutions [34] for the Laplace operator.

In this paper, using his Carleman function, we give an explicit needle sequence in three dimensions. It is written in a closed-form by using an integral involving Mittag-Leffler's function

$$
E_{\alpha}(z)=\sum_{n=0}^{\infty} \frac{z^{n}}{\Gamma(1+\alpha n)}, 0<\alpha \leq 1 .
$$

We never solve integral equations in any sense.

We can summarize the conclusion of this paper in one sentence:

- The regular part of a special Carleman function which has been introduced by Yarmukhamedov yields the desired needle sequence. This makes an explicit link between the probe method and a Carleman function.

It should be pointed out that succeeding to the probe method, the author introduced 
another method which he calls the enclosure method [16]. This method gave us a different way of using the exponential solutions for elliptic equations from Calderón 's way [8] and yielded an explicit extraction formula of the convex hull of the unknown inclusions. This suggests that if one replaces the exponential solutions with any other solutions having a similar property, then one can get several information about unknown inclusions. The paper [20] is just a result in which solutions coming from Mittag-Leffler's function instead of the exponential solutions were employed. The result yielded more than the convex hull and the numerical implementation has been done in [26]. However, this result is restricted to the two dimensional case. From this point of view the result obtained in this paper can be considered as an extension of [20] to three dimensions.

A brief outline of this paper is as follows. In Section 2, we explain what is the probe method and the role of the needle sequence. For the purpose a simple inverse boundary value problem for the Laplace equation is considered. Needless to say, a reader who is familiar with the probe method can skip this section. The main idea is described in Section 3 by considering a two dimensional case. In Section 4 we introduce Yarmukhamedov's fundamental solution for the Laplace operator in three dimensions and show that the regular part of a special version of his fundamental solution yields an explicit needle sequence. In Section 5 we consider how to construct the needle sequence for the Helmholtz equation. We show that the Vekua transform of the regular part of the special fundamental solution for the Laplace operator yields the desired needle sequence. In Appendix, for reader's convenience, we give a direct proof of a theorem established by Yarmukhamedov.

\section{Probe method and needle sequence}

This section is devoted to a reader who is not familiar with the probe method. Recently in $[21,23,24]$ the author reformulated the probe method and further investigated the method itself. It became much simpler than the previous formulation in [13]. In this section, for simplicity of description we restrict ourselves to the case when the unknown discontinuity is coming from cavities.

Let $\Omega$ be a bounded domain of $\mathbf{R}^{m}(m=2,3)$ with a Lipschitz boundary. Let $D$ be a bounded open set of $\Omega$ such that $\bar{D}$ is contained in $\Omega, \partial D$ is Lipschitz, $\Omega \backslash \bar{D}$ is connected. Given $f \in H^{1 / 2}(\partial \Omega)$ let $u \in H^{1}(\Omega \backslash \bar{D})$ be the unique weak solution of the problem

$$
\begin{gathered}
\triangle u=0 \text { in } \Omega \backslash \bar{D}, \\
\frac{\partial u}{\partial \nu}=0 \text { on } \partial D, \\
u=f \text { on } \partial \Omega .
\end{gathered}
$$

The map $\Lambda_{D}: f \longmapsto \partial u /\left.\partial \nu\right|_{\partial \Omega}$ is called the Dirichlet-to-Neumann map. We set $\Lambda_{D}=\Lambda_{0}$ in the case when $D$ is empty.

In short, the probe method is a method of probing inside given material by monitoring the behaviour of the sequence of the energy gap

$$
\left.\int_{\partial \Omega}\left\{\left(\Lambda_{0}-\Lambda_{D}\right)\left(\left.v_{n}\right|_{\partial \Omega}\right)\right\} \bar{v}_{n}\right|_{\partial \Omega} d S
$$


for a specially chosen sequence $\left\{v_{n}\right\}$ of solutions of the governing equation for the background medium $(D=\emptyset)$ which play a role of probe needle.

The method starts with introducing

Definition 2.1. Given a point $x \in \Omega$ we say that a non self-intersecting piecewise linear curve $\sigma$ in $\bar{\Omega}$ is a needle with tip at $x$ if $\sigma$ connects a point on $\partial \Omega$ with $x$ and other points of $\sigma$ are contained in $\Omega$.

We denote by $N_{x}$ the set of all needles with tip at $x$.

Let $\boldsymbol{b}$ be a nonzero vector in $\mathbf{R}^{m}$. Given $x \in \mathbf{R}^{m}, \rho>0$ and $\left.\theta \in\right] 0, \pi$ [ set

$$
C_{x}(\boldsymbol{b}, \theta / 2)=\left\{y \in \mathbf{R}^{m}|(y-x) \cdot \boldsymbol{b}>| y-x|| \boldsymbol{b} \mid \cos (\theta / 2)\right\}
$$

and

$$
B_{\rho}(x)=\left\{y \in \mathbf{R}^{m}|| y-x \mid<\rho\right\}
$$

A set having the form

$$
V=B_{\rho}(x) \cap C_{x}(\boldsymbol{b}, \theta / 2)
$$

for some $\rho, \boldsymbol{b}, \theta$ and $x$ is called a finite cone with vertex at $x$.

Let $G(y)$ be a solution of the Laplace equation in $\mathbf{R}^{m} \backslash\{0\}$ such that for any finite cone $V$ with vertex at 0

$$
\int_{V}|\nabla G(y)|^{2} d y=\infty
$$

Hereafter we fix this $G$.

For the new formulation of the probe method we need the following.

Definition 2.2. Let $\sigma \in N_{x}$. We call the sequence $\xi=\left\{v_{n}\right\}$ of $H^{1}(\Omega)$ solutions of the Laplace equation a needle sequence for $(x, \sigma)$ if it satisfies for any compact set $K$ of $\mathbf{R}^{m}$ with $K \subset \Omega \backslash \sigma$

$$
\lim _{n \longrightarrow \infty}\left(\left\|v_{n}(\cdot)-G(\cdot-x)\right\|_{L^{2}(K)}+\left\|\nabla\left\{v_{n}(\cdot)-G(\cdot-x)\right\}\right\|_{L^{2}(K)}\right)=0 .
$$

The existence of the needle sequence is a consequence of the Runge approximation property for the Laplace equation.

In [21] we clarified the behaviour of the needle sequence on the needle as $n \longrightarrow \infty$. The two lemmas given below are the core of the new formulation of the probe method

Lemma 2.1. Let $x \in \Omega$ be an arbitrary point and $\sigma$ be a needle with tip at $x$. Let $\xi=\left\{v_{n}\right\}$ be an arbitrary needle sequence for $(x, \sigma)$. Then, for any finite cone $V$ with vertex at $x$ we have

$$
\lim _{n \longrightarrow \infty} \int_{V \cap \Omega}\left|\nabla v_{n}(y)\right|^{2} d y=\infty .
$$

Lemma 2.2. Let $x \in \Omega$ be an arbitrary point and $\sigma$ be a needle with tip at $x$. Let $\xi=\left\{v_{n}\right\}$ be an arbitrary needle sequence for $(x, \sigma)$. Then for any point $z \in \sigma$ and open ball $B$ centered at $z$ we have

$$
\lim _{n \rightarrow \infty} \int_{B \cap \Omega}\left|\nabla v_{n}(y)\right|^{2} d y=\infty
$$

From these lemmas we know that one can recover full knowledge of the given needle as the set of all points where the needle sequence for the needle blows up. This means that the needle is realized as a special sequence of harmonic functions without loosing information 
about the geometry of the needle. This is the new point added on the probe method in $[21]$.

Definition 2.3. Given $x \in \Omega$, needle $\sigma$ with tip $x$ and needle sequence $\xi=\left\{v_{n}\right\}$ for $(x, \sigma)$ define

$$
I(x, \sigma, \xi)_{n}=\int_{\partial \Omega}\left\{\left(\Lambda_{0}-\Lambda_{D}\right) \bar{f}_{n}\right\} f_{n} d S, n=1,2, \cdots
$$

where

$$
f_{n}(y)=v_{n}(y), y \in \partial \Omega
$$

$\left\{I(x, \sigma, \xi)_{n}\right\}_{n=1,2, \cdots}$ is a sequence depending on $\xi$ and $\sigma \in N_{x}$. We call the sequence the indicator sequence.

The behaviour of the indicator sequence has two sides. One side is closely related to the function defined below.

Definition 2.4. The indicator function $I$ is defined by the formula

$$
I(x)=\int_{D}|\nabla G(y-x)|^{2} d y+\int_{\Omega \backslash \bar{D}}\left|\nabla w_{x}\right|^{2} d y, x \in \Omega \backslash \bar{D}
$$

where $w_{x}$ is the unique weak solution of the problem:

$$
\begin{gathered}
\triangle w=0 \text { in } \Omega \backslash \bar{D} \\
\frac{\partial w}{\partial \nu}=-\frac{\partial}{\partial \nu}(G(\cdot-x)) \text { on } \partial D \\
w=0 \text { on } \partial \Omega .
\end{gathered}
$$

The function $w_{x}$ is called the reflected solution by $D$.

The following theorem says that

- one can calculate the value of the indicator function at an arbitrary point outside the cavity from $\Lambda_{0}-\Lambda_{D}$;

- the indicator function can not be continued across the boundary of the cavity as a bounded function in the whole domain.

Theorem 2.1.A We have

- (A.1) given $x \in \Omega \backslash \bar{D}$ and needle $\sigma$ with tip at $x$ if $\sigma \cap \bar{D}=\emptyset$, then for any needle sequence $\xi=\left\{v_{n}\right\}$ for $(x, \sigma)$ the sequence $\left\{I(x, \sigma, \xi)_{n}\right\}$ converges to the indicator function $I(x)$

- (A.2) for each $\epsilon>0$

$$
\sup _{\operatorname{dist}(x, D)>\epsilon} I(x)<\infty
$$

- (A.3) for any point $a \in \partial D$

$$
\lim _{x \rightarrow a} I(x)=\infty
$$

Since mathematically Theorem 2.1.A is enough for establishing a reconstruction formula of the cavities, in the previous applications of the probe method [13] we did not consider the natural question 
- what happens on the indicator sequence when the tip of the needle is just located on the boundary of cavities, inside or passing through the cavities?

However, in practice the tip of the needle cannot move forward with infinitely small step and therefore in the scanning process with needle there is a possibility of skipping the unknown boundary of cavities, entering inside or passing through the cavities. So for the practical use of the probe method we have to clarify the behaviour of the indicator sequence in those cases. The answer to this question is

Theorem 2.1.B Let $x \in \Omega$ and $\sigma \in N_{x}$. If $x \in \Omega \backslash \bar{D}$ and $\sigma \cap D \neq \emptyset$ or $x \in \bar{D}$, then for any needle sequence $\xi=\left\{v_{n}\right\}$ for $(x, \sigma)$ we have $\lim _{n \rightarrow \infty} I(x, \sigma, \xi)_{n}=\infty$.

See Figure 1 for typical situations.

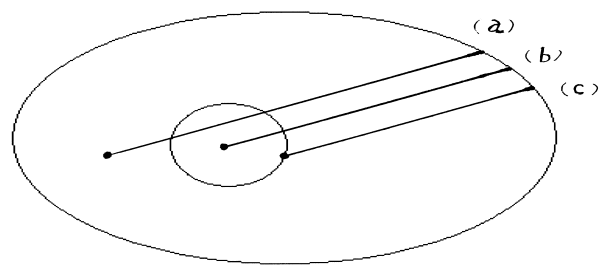

Figure 1: (a) $x \in \Omega \backslash \bar{D}$ and $\sigma \cap D \neq \emptyset$. (b) $x \in D$. (c) $x \in \partial D$.

These two theorems are essentially a special case of the results in [21]. Finally we note that, as a corollary of Theorems $2.1 \mathrm{~A}$ and B one gets a characterization of $\bar{D}$.

Corollary 2.4 A point $x \in \Omega$ belongs to $\Omega \backslash \bar{D}$ if and only if there exists a needle $\sigma$ with tip at $x$ and needle sequence $\xi$ for $(x, \sigma)$ such that the indicator sequence $\left\{I(x, \sigma, \xi)_{n}\right\}$ is bounded from above.

Needless to say, this automatically gives a uniqueness theorem too.

\section{Main idea: a new role of a Carleman function}

We think that the reader now understand the basic of the probe method and the role of the needle sequence. So the next problem is how to construct the needle sequence. 
There are several points for the meaning of the 'construction'. It is well known that one can construct the needle sequence by solving infinitely many first kind integral equations in the sense of minimum norm solutions. The minimum norm solutions are given by a combination of the Tikhonov regularization method and Morozov discrepancy principle(see [29] for these concepts). However, from our point of view this does not yield an explicit needle sequence. One can also point out that the determination of the regularization parameter via the Morozov discrepancy principle itself is a nonlinear problem and seems impossible to find the parameter explicitly.

So how can one construct the needle sequence explicitly? In this and following sections we always consider a geometrically simplest needle described in

Definition 3.1 A needle with tip at $x$ is called a straight needle with tip at $x$ directed to $\omega$ if the needle is given by $l_{x}(\omega) \cap \bar{\Omega}$ where

$$
l_{x}(\omega)=\{x+t \omega \mid 0 \leq t<\infty\} .
$$

In two dimensions in [25] we constructed an explicit needle sequence for $G$ given by

$$
G(y)=\frac{1}{y_{1}+i y_{2}} .
$$

Note that this $G$ satisfies (2.1) for any finite cone $V$ with vertex at 0 .

For the construction we made use of a well known idea in complex function theory:

$$
\begin{gathered}
\frac{1}{y-x}=\frac{1}{\left(y-x^{\prime}\right)-\left(x-x^{\prime}\right)} \\
=\frac{1}{\left(y-x^{\prime}\right)\left(1-\frac{x-x^{\prime}}{y-x^{\prime}}\right)} \\
=\sum_{m=0}^{\infty} \frac{\left(x-x^{\prime}\right)^{m}}{\left(y-x^{\prime}\right)^{m+1}}
\end{gathered}
$$

provided $x^{\prime} \approx x$ and $x^{\prime}$ is located on a needle with tip at $x$. Note that we used the same symbol for the complex number $z_{1}+i z_{2}$ corresponding to a point $z=\left(z_{1}, z_{2}\right)^{T}$.

However, in general we have to do this type of procedure many times and therefore the resulted sequence is quite involved.

In this section, we give a different and extremely simple needle sequence and explain the idea behind.

Definition 3.2. Given a unit vector $\omega=\left(\omega_{1}, \omega_{2}\right)^{T}$ in $\mathbf{R}^{2}$ and $\left.\left.\alpha \in\right] 0,1\right]$ define

$$
v(y ; \alpha, \tau, \omega)=-\frac{E_{\alpha}(\tau y \bar{\omega})-1}{y}, \tau>0 .
$$

This is a harmonic function in the whole plane. Note also that $y \cdot\left(\omega+i \omega^{\perp}\right)=y \bar{\omega}$ where $\omega^{\perp}=\left(-\omega_{2}, \omega_{1}\right)$.

Theorem 3.1 Let $x \in \Omega$ and $\sigma$ be a straight needle with tip at $x$ directed to $\omega$. The sequence $\left\{\left.v\left(\cdot-x ; \alpha_{n}, \tau_{n}, \omega\right)\right|_{\Omega}\right\}$ is a needle sequence for $(x, \sigma)$ where $\alpha_{n}$ and $\tau_{n}$ are suitably chosen sequences and satisfy 
- $0<\alpha_{n} \leq 1, \tau_{n}>0$

- $\alpha_{n} \longrightarrow 0$ and $\tau_{n} \longrightarrow \infty$ as $n \longrightarrow \infty$.

Proof. For each fixed $\alpha$ we have, as $\tau \longrightarrow \infty$,

$$
v(\cdot-x ; \alpha, \tau, \omega) \longrightarrow G(\cdot-x)
$$

uniformly for $y$ in any compact subset of $\mathbf{R}^{2} \backslash \overline{C_{x}(\pi \alpha / 2, \omega)}$. This is a direct consequence of the well known asymptotic behaviour of Mittag-Leffler's function [6, 11]: as $|z| \longrightarrow \infty$ with $\pi \alpha / 2+\epsilon \leq|\arg z| \leq \pi$

$$
E_{\alpha}(z)=-\frac{1}{z \Gamma(1-\alpha)}+O\left(\frac{1}{|z|^{2}}\right)
$$

where $\alpha$ and $\epsilon$ are fixed and satisfies $0<\alpha<1$ and $0<\epsilon<\pi-\pi \alpha / 2$.

The choice of $\left\{\alpha_{n}\right\}$ and $\left\{\tau_{n}\right\}$ depend on that of an exhaustion for $\Omega \backslash \sigma$. More precisely let $\left\{O_{n}\right\}$ be a sequence of open subsets of $\Omega \backslash \sigma$ such that for all $\bar{O}_{n} \subset O_{n+1} ; \cup_{n} O_{n}=\Omega \backslash \sigma$. We call this sequence an exhaustion for $\Omega \backslash \sigma$. Let $\left\{\epsilon_{n}\right\}$ be an arbitrary sequence of positive numbers such that $\epsilon_{n} \longrightarrow 0$ as $n \longrightarrow \infty$. The choice of $\left\{\alpha_{n}\right\}$ and $\left\{\tau_{n}\right\}$ can be done as follows. Since $\bar{O}_{1} \subset \Omega \backslash \sigma$, one can choose a small $\left.\alpha \in\right] 0,1[$ in such a way that $\bar{O}_{1}$ is contained in the set $\mathbf{R}^{2} \backslash \overline{C_{x}(\pi \alpha / 2, \omega)}$. Set $\alpha_{1}=\alpha$. Since $v\left(y-x ; \alpha_{1}, \tau, \omega\right)$ converges to $G(y-x)$ in $H^{1}\left(\Omega_{1}\right)$ as $\tau \longrightarrow \infty$, one can find a large $\tau$ in such a way that $\left\|v\left(\cdot-x ; \alpha_{1}, \tau, \omega\right)-G(\cdot-x)\right\|_{H^{1}\left(O_{1}\right)}<\epsilon_{1}$. Set $\tau_{1}=\tau$. For $\alpha_{2}, \cdots$ and $\tau_{2}, \cdots$ we do a similar procedure. Then the obtained sequence satisfies the desired property.

We see that $v\left(y-x ; \alpha_{n}, \tau_{n}, \omega\right)$ takes the special value at the tip $x$ of the needle:

$$
\left.v\left(y-x ; \alpha_{n}, \tau_{n}, \omega\right)\right|_{y=x}=-\frac{\tau_{n} \bar{\omega}}{\Gamma\left(1+\alpha_{n}\right)}
$$

and thus this yields

$$
\lim _{n \longrightarrow \infty} \frac{\left.v\left(y-x ; \alpha_{n}, \tau_{n}, \omega\right)\right|_{y=x}}{\tau_{n}}=-\bar{\omega} .
$$

Therefore the leading term of the asymptotic behaviour of this special needle sequence at the tip $x$ of needle $\sigma$ does not depend on the choice of $\left\{\alpha_{n}\right\}$ and is uniquely determined by $\left\{\tau_{n}\right\}$.

However, the point of this section is not the statement of Theorem 3.1 itself. The main point is how to find this simple form. For simplicity let $x=0$.

First the function $G(y)$ given by (3.1) is a fundamental solution for the operator $\bar{\partial}$ (ignoring multiplying a constant, hereafter same). Since $E_{\alpha}(0)=1$, the function

$$
\frac{E_{\alpha}(\tau y \bar{\omega})}{y}
$$

is also a fundamental solution for operator $\bar{\partial}$. The point is: this fundamental solution becomes small outside a sector as $\tau \longrightarrow \infty[6,11]$. Since we can write

$$
\frac{E_{\alpha}(\tau y \bar{\omega})}{y}=G(y)+\frac{\left.E_{\alpha}(\tau y \bar{\omega})\right)-1}{y}
$$


this means that the outside the sector

$$
G(y) \approx-\frac{E_{\alpha}(\tau y \bar{\omega})-1}{y} .
$$

Note that this right-hand side is just coming from the regular part of (3.4).

The function (3.4) has been appeared in Yarmukhamedov's work [35] to give an explicit formula of the value of the solution of the Cauchy problem in a special domain

for $\bar{\partial}$. Such a type of fundamental solution for some operator is called the Carleman function (for the operator) and the formula is called a Carleman-type formula. See also [1] for Carleman-type formulae in complex function theory and $[2,18]$ for the stationary Schrödinger equation.

So now the principle of the choice of needle sequence is clear. In particular, in three dimensions the principle is: choose $(-1)$ times the regular part of a suitable Carleman function (for the Laplace operator).

This principle is the conclusion of this paper and gives a new role for the Carleman function. We found a direct link between the Carleman function and the probe method. Needless to say, it is different from the idea in existing author's previous application [17] of the Carleman function to an inverse boundary value problem in unbounded domain.

In the next section we consider the three dimensional case more precisely.

\section{Yarmukhamedov's fundamental solution and nee- dle sequence in three dimensions}

The following is taken from [34]; however, therein the proof is not given. For reader's convenience we gave a direct proof of this theorem in Appendix.

Theorem 4.1 Let $\lambda \geq 0$. Let $K(w)$ be an entire function such that

- $K(w)$ is real for real $w$

- $K(0)=1$

- for each $R>0$ and $m=0,1,2$

$$
\sup _{|R e w|<R}\left|K^{(m)}(w)\right| e^{\lambda|I m w|}<\infty .
$$

Define

$$
-2 \pi^{2} \Phi(x)=\frac{1}{2} \int_{0}^{\infty} \operatorname{Im}\left(\frac{K(w)}{w}\right) \frac{e^{\lambda u}+e^{-\lambda u}}{\sqrt{\left|x^{\prime}\right|^{2}+u^{2}}} d u .
$$

where $w=x_{3}+i \sqrt{\left|x^{\prime}\right|^{2}+u^{2}}$ and $x^{\prime}=\left(x_{1}, x_{2}\right)$. Then one has the expression

$$
\Phi(x)=\frac{e^{i \lambda|x|}}{4 \pi|x|}+H(x)
$$

where $H$ is $C^{2}$ in the whole space and satisfies

$$
\triangle H(x)+\lambda^{2} H(x)=0 \text { in } \mathbf{R}^{3}
$$


and therefore $\Phi$ satisfies

$$
\triangle \Phi(x)+\lambda^{2} \Phi(x)+\delta(x)=0 \text { in } \mathbf{R}^{3} .
$$

Hereafter we set $\Phi=\Phi_{K}$ to denote the dependence on $K$ and consider only the case when $\lambda=0$. In this case (3.2) ensures that $K(w)=E_{\alpha}(\tau w)$ with $\tau>0$ satisfies (4.1). Yarmukhamedov [36] established that the function $\Phi_{K}$ for this $K$ with a fixed $\alpha$ is a Carleman function (for the Laplace equation).

Definition 4.1 Given two unit vectors $\vartheta_{1}$ and $\vartheta_{2}$ in three dimensions and $\left.\alpha \in\right] 0,1$ ] define

$$
v\left(y ; \alpha, \tau, \vartheta_{1}, \vartheta_{2}\right)=-\left\{\Phi_{K}\left(y \cdot \vartheta_{1}, y \cdot \vartheta_{2}, y \cdot\left(\vartheta_{1} \times \vartheta_{2}\right)\right)-\frac{1}{4 \pi|y|}\right\}, \tau>0
$$

where $K(w)=E_{\alpha}(\tau w)$. From Theorem 4.1 for $\lambda=0$ one knows that this function of $y$ is nothing but the regular part of $\Phi_{K}$ and harmonic in the whole space.

In this section we prove

Theorem 4.2 Let $x \in \Omega$ and $\sigma$ be a straight needle with tip at $x$ directed to $\omega=\vartheta_{1} \times \vartheta_{2}$. Then the sequence $\left\{\left.v\left(\cdot-x ; \alpha_{n}, \tau_{n}, \vartheta_{1}, \vartheta_{2}\right)\right|_{\Omega}\right\}$ is a needle sequence for $(x, \sigma)$ with

$$
G(y)=\frac{1}{4 \pi|y|}
$$

where $\alpha_{n}$ and $\tau_{n}$ are suitably chosen sequences and satisfy

- $0<\alpha_{n}<1, \tau_{n}>0$

- $\alpha_{n} \longrightarrow 0$ and $\tau_{n} \longrightarrow \infty$.

Proof. First we prove that, for each fixed $\alpha$ as $\tau \longrightarrow \infty$

$$
v\left(\cdot-x ; \alpha, \tau, \vartheta_{1}, \vartheta_{2}\right) \longrightarrow G(\cdot-x)
$$

uniformly for $y$ in any compact subset of $\mathbf{R}^{3} \backslash \overline{C_{x}(\pi \alpha / 2, \omega)}$.

Given $\eta \in] 0, \pi[, r>0$ let $\gamma(\eta, r)$ denote the contour that originates at $\infty$, runs in toward the origin just above the half line $z=\rho e^{-i \eta}, \rho \geq r$, the part of the circle $z=r e^{i \theta},|\theta| \leq \eta$, the origin counterclockwise and then returns to $\infty$ just above the half line $z=\rho e^{i \eta}, \rho \geq r$. The contour $\gamma$ splits the complex plane into the two simply connected infinite domains $D^{-}(\eta, r)$ and $D^{+}(\eta, r)$ lying, respectively to the left and the right of $\gamma(\eta, r)$.

From Mittag-Leffler's integral representation (p. 206 of [6]) and analytic continuation, it follows that, for any $\eta \in] \pi / 2, \pi[$

$$
E_{\alpha}(z)=\frac{1}{2 \pi i} \int_{\gamma(\eta, r)} \frac{\zeta^{\alpha-1} e^{\zeta}}{\zeta^{\alpha}-z} d \zeta, z \in D^{-}\left(\alpha \eta, r^{\alpha}\right) .
$$

Let $\tau>0$. We see that: if $z \in D^{-}\left(\alpha \eta, r^{\alpha}\right) \backslash B_{r^{\alpha}}(0)$, then $\tau z \in D^{-}\left(\alpha \eta, r^{\alpha}\right)$; if $z \in$ $D^{-}\left(\alpha \eta, r^{\alpha}\right)$, then so is $\bar{z}$. And it should be pointed out that $E_{\alpha}(z)$ is real for real $z$. From these we have, for all $z \in D^{-}\left(\alpha \eta, r^{\alpha}\right) \backslash B_{r^{\alpha}}(0)$,

$$
\frac{1}{\operatorname{Im} z} \operatorname{Im}\left(\frac{E_{\alpha}(\tau z)}{z}\right)=-\frac{1}{2 \pi i|z|^{2}} \int_{\gamma(\eta, r)} \frac{\left(\zeta^{\alpha}-2 \tau \operatorname{Re} z\right) \zeta^{\alpha-1} e^{\zeta}}{\left(\zeta^{\alpha}-\tau z\right)\left(\zeta^{\alpha}-\tau \bar{z}\right)} d \zeta
$$


Here we restrict the interval where $\eta$ belongs to $\eta \in] \pi / 2, \pi /(2 \alpha)[$. Let $\theta$ satisfy $\alpha \eta<\theta<\pi / 2$. Then we have, for all $w \in D^{-}\left(\theta, r^{\alpha}\right) \backslash B_{r^{\alpha}}(0)$ and $\tau>1$,

$$
\inf _{\zeta \in \gamma(\eta, r)}\left|\zeta^{\alpha}-\tau w\right| \geq C \tau|w|
$$

where

$$
C=\min \left\{1-\frac{1}{\tau}, \sin (\theta-\alpha \eta)\right\} .
$$

This can be proved as follows. It suffices to consider the case when $\theta \leq \arg w \equiv \varphi \leq \pi$. We divide the case into two subcases. First let $\theta \leq \varphi \leq \alpha \eta+\pi / 2$. In this case the circle $|z-w|=|w| \sin (\varphi-\alpha \eta)$ has a single common point with the half line $z=k e^{i \alpha \eta}, r^{\alpha} \leq$ $k<\infty$. Thus this yields

$$
\inf _{\zeta \in \gamma(\eta, r)}\left|\zeta^{\alpha}-w\right|=|w| \sin (\varphi-\alpha \eta) .
$$

Since $\theta-\alpha \eta \leq \varphi-\alpha \eta \leq \pi / 2$, we obtain

$$
\inf _{\zeta \in \gamma(\eta, r)}\left|\zeta^{\alpha}-w\right| \geq|w| \sin (\theta-\alpha \eta) .
$$

Next consider the case $\alpha \eta+\pi / 2<\varphi \leq \pi$. A simple geometrical observation yields

$$
\inf _{\zeta \in \gamma(\eta, r)}\left|\zeta^{\alpha}-w\right|=\left|w-r^{\alpha} e^{i \alpha \eta}\right| \geq|w|-r^{\alpha} .
$$

Now let $\tau>1$. Then $\tau w$ still belongs to $D^{-}\left(\theta, r^{\alpha}\right) \backslash B_{r^{\alpha}}(0)$ and having the same arg as $w$. Therefore, inserting $\tau w$ instead of $w$ into (4.7) we obtain

$$
\inf _{\zeta \in \gamma(\eta, r)}\left|\zeta^{\alpha}-\tau w\right| \geq \tau|w|-r^{\alpha} \geq(\tau-1)|w| .
$$

Now a combination of (4.6) and (4.8) gives (4.5).

Now let $y$ satisfy

$$
(y-x) \cdot \omega \leq|y-x| \cos \theta
$$

and

$$
r^{\alpha} \leq|y-x| \leq R^{\alpha}
$$

Set $w=(y-x) \cdot \omega+i \sqrt{\left|(y-x) \cdot \vartheta_{1}\right|^{2}+\left|(y-x) \cdot \vartheta_{2}\right|^{2}+u^{2}}$. This $w$ belongs to $D^{-}\left(\theta, r^{\alpha}\right) \backslash$ $B_{r^{\alpha}}(0)$. Therefore from (4.4) and (4.5) we obtain

$$
\left|\frac{1}{\operatorname{Im} w} \operatorname{Im}\left(\frac{E_{\alpha}(\tau w)}{w}\right)\right| \leq \frac{C}{2 \pi \tau\left(r^{2 \alpha}+u^{2}\right)^{2}} \int_{\gamma(\eta, r)}\left(|\zeta|^{\alpha}+2 R^{\alpha}\right)|\zeta|^{\alpha-1} e^{\operatorname{Re} \zeta}|d \zeta| .
$$

The integral in this right hand side is absolutely convergent since $\pi / 2<\eta<\pi$. Therefore, we have

$$
\Phi_{K}\left((y-x) \cdot \vartheta_{1},(y-x) \cdot \vartheta_{2},(y-x) \cdot \omega\right)=O\left(\frac{1}{\tau}\right)
$$

as $\tau \longrightarrow \infty$ and the estimate is uniform for $y-x$ with (4.9) and (4.10) for each fixed $\theta$, $\eta, r, R$ and $\alpha$.

It is easy to see that, choosing suitable $\theta, \eta, r$, a large fixed $R$ and $\alpha$, one can generate an exhaustion of the domain $\Omega \backslash \sigma$. Therefore from the principle explained in Section 3 
we conclude that the sequence $\left\{\left.v\left(\cdot-x ; \alpha_{n}, \tau_{n}, \vartheta_{1}, \vartheta_{2}\right)\right|_{\Omega}\right\}$ converges to $G(y)$ in $L_{\text {loc }}^{2}(\Omega \backslash$ $\sigma)$ for suitably chosen sequences $\left\{\alpha_{n}\right\}$ and $\left\{\tau_{n}\right\}$. Then, a standard argument gives the convergence in $H_{\text {loc }}^{1}(\Omega \backslash \sigma)$.

Remark 4.1. The proof presented here can be considered as a minor modification of that of Lemma in [36]. Anyway the new point that should be emphasized is the relationship between the construction of a needle sequence and a Carleman function.

Definition 4.2. We call the needle sequence $\left\{\left.v\left(\cdot-x ; \alpha_{n}, \tau_{n}, \vartheta_{1}, \vartheta_{2}\right)\right|_{\Omega}\right\}$ given in Theorem 4.2 a standard needle sequence for $(x, \sigma)$ for the Laplace equation.

Choosing $K(w)=1$ in $\Phi_{K}$, we have

$$
\Phi_{1}(y)=\frac{1}{4 \pi|y|} .
$$

This gives the expression:

$$
2 \pi^{2} v\left(y ; \alpha, \tau, \vartheta_{1}, \vartheta_{2}\right)=\int_{0}^{\infty} \operatorname{Im}\left(\frac{E_{\alpha}(\tau w)-1}{w}\right) \frac{d u}{\sqrt{\left|y \cdot \vartheta_{1}\right|^{2}+\left|y \cdot \vartheta_{2}\right|^{2}+u^{2}}}
$$

where

$$
w=y \cdot \omega+i \sqrt{\left|y \cdot \vartheta_{1}\right|^{2}+\left|y \cdot \vartheta_{2}\right|^{2}+u^{2}} .
$$

The advantage of the standard needle sequence is that one can exactly know the precise values in a closed-form on the needle and its linear extension.

Theorem 4.3 Let $\omega=\vartheta_{1} \times \vartheta_{2}$. We have:

(1) if $y=x+s \omega$ with $s \neq 0$, then

$$
v\left(y-x ; \alpha, \tau, \vartheta_{1}, \vartheta_{2}\right)=\frac{E_{\alpha}(\tau s)-1}{4 \pi s} ;
$$

(2) if $y=x$, then

$$
\left.v\left(y-x ; \alpha, \tau, \vartheta_{1}, \vartheta_{2}\right)\right|_{y=x}=\frac{\tau}{4 \pi \Gamma(1+\alpha)} .
$$

Proof.

From (4.11) we have the expression of $v\left(y-x ; \alpha, \tau, \vartheta_{1}, \vartheta_{2}\right)$ in both cases $(1)$ and $(2)$ :

$$
2 \pi^{2} v\left(y-x ; \alpha, \tau, \vartheta_{1}, \vartheta_{2}\right)=\int_{0}^{\infty} \operatorname{Im}\left(\frac{E_{\alpha}\left(\tau\left(x_{3}+i u\right)\right)-1}{x_{3}+i u}\right) \frac{d u}{u}
$$

here we set $x_{3}=(y-x) \cdot \omega$ for simplicity of description. One can write

$$
\int_{0}^{\infty} \operatorname{Im}\left(\frac{E_{\alpha}\left(\tau\left(x_{3}+i u\right)\right)-1}{x_{3}+i u}\right) \frac{d u}{u}=\frac{1}{2 i} \lim _{\epsilon \downarrow 0}\left(\int_{-i / \epsilon}^{-i \epsilon}+\int_{i \epsilon}^{i / \epsilon}\right) \frac{E_{\alpha}\left(\tau\left(x_{3}+\zeta\right)\right)-1}{\left(x_{3}+\zeta\right) \zeta} d \zeta .
$$

First consider the case when $x_{3} \neq 0$. Then $\zeta=-x_{3}$ is a removable singular point for the integrand. Therefore, from the Cauchy's integral theorem and (3.2) we see that the right hand side of (4.15) becomes

$$
\frac{1}{2 i} \lim _{\epsilon \downarrow 0} \int_{\zeta=\epsilon e^{i \theta},|\theta-\pi| \leq \pi / 2} \frac{E_{\alpha}\left(\tau\left(x_{3}+\zeta\right)\right)-1}{\left(x_{3}+\zeta\right) \zeta} d \zeta=\frac{\pi}{2} \frac{E_{\alpha}\left(\tau x_{3}\right)-1}{x_{3}} .
$$


This together with (4.14) and $(y-x) \cdot \omega=s$ for $y=x+s \omega$ yields (4.12).

A similar computation in the case when $x_{3}=0$ yields (4.13).

The formula (4.13) corresponds to (3.3).

Remark 4.2. From (4.12), (4.13) and the trivial estimate

$$
E_{\alpha}(x)-1>\frac{x^{n}}{\Gamma(1+\alpha n)}, x>0, n=1,2, \cdots
$$

we see that the standard needle sequence on the needle blows up in a point wise sense: for all points $y$ on $l_{x}(\omega)$ (see Definition 3.1) $\lim _{n \longrightarrow \infty} v\left(y-x ; \alpha_{n}, \tau_{n}, \vartheta_{1}, \vartheta_{2}\right)=+\infty$.

Remark 4.3. We can know also the values of $\nabla v\left(y-x ; \alpha, \tau, \vartheta_{1}, \vartheta_{2}\right)$ on the line $y=$ $x+s \omega(-\infty<s<\infty)$ with $\omega=\vartheta_{1} \times \vartheta_{2}$. Define

$$
I_{1}(\rho, s)=\int_{1}^{\infty} \operatorname{Im}\left(\frac{E_{\alpha}\left(\tau\left(s+i \sqrt{\rho+u^{2}}\right)\right)}{s+i \sqrt{\rho+u^{2}}}\right) \frac{d u}{\sqrt{\rho+u^{2}}}, \rho>-1,-\infty<s<\infty
$$

and

$$
I_{2}(\rho, s)=\int_{0}^{1} \operatorname{Im}\left(\frac{E_{\alpha}\left(\tau\left(s+i \sqrt{\rho+u^{2}}\right)\right)}{s+i \sqrt{\rho+u^{2}}}\right) \frac{d u}{\sqrt{\rho+u^{2}}}, 0<\rho<1,-\infty<s<\infty .
$$

Using (3.2) and the asymptotic expansions of the derivatives of Mittag-Leffler's function outside the sector $|\arg z| \leq \pi \alpha / 2$, we conclude that $I_{1}(\rho, s)$ is a smooth function of $(\rho, s)$. Following the argument in Appendix A.2 we have

$$
\begin{gathered}
I_{2}(\rho, s)=-\int_{0}^{1} \frac{d u}{\rho+s^{2}+u^{2}} \\
+\sum_{n=1}^{\infty} \frac{\tau^{n+1}}{\Gamma(1+\alpha(n+1))} \sum_{j \text { is odd }} \frac{n !}{j !(n-j) !} s^{n-j}(-1)^{(j-1) / 2} \int_{0}^{1}\left(\rho+u^{2}\right)^{(j-1) / 2} d u .
\end{gathered}
$$

Since the second term is smooth for $(\rho, s) \in \mathbf{R}^{2}$, this yields that the function

$$
I_{3}(\rho, s) \equiv I_{2}(\rho, s)+\int_{0}^{1} \frac{d u}{\rho+s^{2}+u^{2}}
$$

has a smooth extension to $\mathbf{R}^{2}$. Since

$$
\int_{0}^{\infty} \operatorname{Im}\left(\frac{1}{s+i \sqrt{\rho+u^{2}}}\right) \frac{d u}{\sqrt{\rho+u^{2}}}=-\int_{0}^{\infty} \frac{d u}{\rho+s^{2}+u^{2}}
$$

we have the decomposition of (4.11):

$$
2 \pi^{2} v\left(y ; \alpha, \tau, \vartheta_{1}, \vartheta_{2}\right)=I_{1}(\rho, s)+I_{3}(\rho, s)
$$

where $\rho=\left|y \cdot \vartheta_{1}\right|^{2}+\left|y \cdot \vartheta_{2}\right|^{2}$ and $s=y \cdot \omega$. Therefore we obtain

$$
2 \pi^{2} \nabla v\left(y ; \alpha, \tau, \vartheta_{1}, \vartheta_{2}\right)=\frac{\partial}{\partial s}\left(I_{1}+I_{3}\right) \omega+2 \frac{\partial}{\partial \rho}\left(I_{1}+I_{3}\right)\left\{\left(y \cdot \vartheta_{1}\right) \vartheta_{1}+\left(y \cdot \vartheta_{2}\right) \vartheta_{2}\right\}
$$


This together with (4.12) and (4.16) yields that for $y=x+s \omega$ with $s \neq 0$

$$
\nabla v\left(y-x ; \alpha, \tau, \vartheta_{1}, \vartheta_{2}\right)=\frac{d}{d s}\left\{\frac{E_{\alpha}(\tau s)-1}{4 \pi s}\right\} \omega .
$$

Moreover, letting $s \longrightarrow 0$, we have

$$
\left.\nabla v\left(y-x ; \alpha, \tau, \vartheta_{1}, \vartheta_{2}\right)\right|_{y=x}=\frac{\tau^{2}}{4 \pi \Gamma(1+2 \alpha)} \omega .
$$

Therefore $\nabla v$ on the line $y=x+s \omega(-\infty<s<\infty)$ is parallel to the direction of the line. In particular, using the power series expansion of Mittag-Leffler's function, we see that $\nabla v\left(y-x ; \alpha, \tau, \vartheta_{1}, \vartheta_{2}\right) \cdot \omega>0$ and $\lim _{n \rightarrow \infty} \nabla v\left(y-x ; \alpha_{n}, \tau_{n}, \vartheta_{1}, \vartheta_{2}\right) \cdot \omega=+\infty$ on the set $l_{x}(\omega)$.

\section{$5 \quad$ Needle sequence for Helmholtz equation and Vekua transform}

The needle sequence for the Helmoltz equation can be defined as same as the Laplace equation.

Let $G(y)$ be a solution of the Helmholtz equation $\triangle v+\lambda^{2} v=0$ in $\mathbf{R}^{3} \backslash\{0\}$ such that for any finite cone $V$ with vertex at $0 G$ satisfies (2.1). Hereafter we fix $G$.

Definition 5.1. Let $\sigma \in N_{x}$. We call the sequence $\xi=\left\{v_{n}\right\}$ of $H^{1}(\Omega)$ solutions of the Helmholtz equation $\Delta v+\lambda^{2} v=0$ a needle sequence for $(x, \sigma)$ for the Helmholtz equation if it satisfies, for any compact set $K$ of $\mathbf{R}^{3}$ with $K \subset \Omega \backslash \sigma$

$$
\lim _{n \longrightarrow \infty}\left(\left\|v_{n}(\cdot)-G(\cdot-x)\right\|_{L^{2}(K)}+\left\|\nabla\left\{v_{n}(\cdot)-G(\cdot-x)\right\}\right\|_{L^{2}(K)}\right)=0 .
$$

Remark 5.1. Any needle sequences for the Helmholtz equation with an arbitrary $\lambda$ blows up on the needle in the sense of Lemmas 2.1 and 2.2. See [21] for the proof.

The existence of the needle sequence for the Helmholtz equation has been ensured under the additional condition on $\lambda$ (see Appendixes of $[14,21]$ for the proof):

- $\lambda^{2}$ is not a Dirichlet eigenvalue of $-\triangle$ in $\Omega$.

However, the proof therein does not give us any explicit form of a needle sequence. Since the needle sequence for the Helmholtz equation plays a central role in the probe method applied to inverse obstacle scattering problems (see [14, 21] again and [24] for a recent application), it is quite important to obtain an explicit one.

In this section we give explicit needle sequences for the Helmholtz equation for all straight needles and all $\lambda(>0)$. There is no additional condition on $\lambda$. For the construction we do not make use of Theorem 4.1. Mittag-Leffler's function with $\alpha<1$ does not satisfy the condition (4.1) when $\lambda>0$. Therefore one can not substitute $K(w)=E_{\alpha}(\tau w)$ into (4.2) to construct a needle sequence for the Helmoltz equation $\triangle u+\lambda^{2} u=0$ in three dimensions. In this section, instead of (4.2) we employ an idea of making use of a transformation introduced by Vekua $[30,31]$.

Let $\lambda>0$. The Vekua transform $v \longmapsto T_{\lambda} v$ in three dimensions takes the form

$$
T_{\lambda} v(y)=v(y)-\frac{\lambda|y|}{2} \int_{0}^{1} v(t y) J_{1}(\lambda|y| \sqrt{1-t}) \sqrt{\frac{t}{1-t}} d t
$$


where $J_{1}$ stands for the Bessel function of order 1 . The important property of this transform is: if $v$ is harmonic in the whole space, then $T_{\lambda} v$ is a solution of the Helmholtz equation $\triangle u+\lambda^{2} u=0$ in the whole space.

Definition 5.2. Given two unit vectors $\vartheta_{1}$ and $\vartheta_{2}$ in three dimensions and $\left.\left.\alpha \in\right] 0,1\right]$ define

$$
v^{\lambda}\left(y ; \alpha, \tau, \vartheta_{1}, \vartheta_{2}\right)=T_{\lambda} v\left(y ; \alpha, \tau, \vartheta_{1}, \vartheta_{2}\right), \tau>0 .
$$

This function of $y$ satisfies the Helmholtz equation $\triangle u+\lambda^{2} u=0$ in the whole space.

Theorem 5.1 Let $x \in \Omega$ and $\sigma$ be a straight needle with tip at $x$ directed to $\omega=\vartheta_{1} \times \vartheta_{2}$. Then the sequence $\left\{\left.v^{\lambda}\left(\cdot-x ; \alpha_{n}, \tau_{n}, \vartheta_{1}, \vartheta_{2}\right)\right|_{\Omega}\right\}$ is a needle sequence for $(x, \sigma)$ for the Helmholtz equation with $G=G_{\lambda}$ given by

$$
G_{\lambda}(y)=\operatorname{Re}\left(\frac{e^{i \lambda|y|}}{4 \pi|y|}\right)
$$

where $\alpha_{n}$ and $\tau_{n}$ are suitably chosen sequences and satisfy

- $0<\alpha_{n}<1, \tau_{n}>0$

- $\alpha_{n} \longrightarrow 0$ and $\tau_{n} \longrightarrow \infty$.

Proof. It suffices to prove the theorem in the case when $x=0, \vartheta_{1}=(1,0,0)^{T}, \vartheta_{2}=$ $(0,1,0)^{T}$ and thus $\omega=(0,0,1)^{T}$.

First we show that for each fixed $\alpha$ as $\tau \longrightarrow \infty$ the function $v^{\lambda}\left(y ; \alpha, \tau, \vartheta_{1}, \vartheta_{2}\right)$ converges to the function $v(y)$ defined by

$$
v(y)=\Phi_{1}(y)-\frac{\lambda|y|}{2} \int_{0}^{1} \Phi_{1}(t y) J_{1}(\lambda|y| \sqrt{1-t}) \sqrt{\frac{t}{1-t}} d t, y \neq 0
$$

uniformly for $y$ in any compact subset of $\mathbf{R}^{3} \backslash \overline{C_{0}(\pi \alpha / 2, \omega)}$.

From Theorem 4.2 we have, $\Phi_{K}(y)$ with $K(z)=E_{\alpha}(\tau z)$ converges to 0 uniformly for $y$ in any compact subset of $\mathbf{R}^{3} \backslash \overline{C_{0}(\pi \alpha / 2, \omega)}$ as $\tau \longrightarrow \infty$. Since $\Phi_{1}(y)-v\left(y ; \alpha, \tau, \vartheta_{1}, \vartheta_{2}\right)=$ $\Phi_{K}(y)$, it suffices to prove that the following function of $y$

$$
R(y ; \alpha, \tau)=\int_{0}^{1} \Phi_{K}(t y) J_{1}(\lambda|y| \sqrt{1-t}) \sqrt{\frac{t}{1-t}} d t, y \neq 0
$$

converges to 0 uniformly for $y$ in any compact subset of $\mathbf{R}^{3} \backslash \overline{C_{0}(\pi \alpha / 2, \omega)}$.

Let $\eta \in] \pi / 2, \pi[$. Let $r$ and $R$ be arbitrary positive numbers with $r<R$. Let $\theta \in$ ] $\alpha \eta, \pi / 2\left[\right.$. Let $y$ satisfy (4.9) and (4.10) with $x=0$ and $\omega=(0,0,1)^{T}$. Then the non zero complex number

$$
w=y_{3}+i \sqrt{y_{1}^{2}+y_{2}^{2}+u^{2}}, u \geq 0
$$

satisfies $\theta \leq|\arg w| \leq \pi$. Then we see that, for all $\tau>0 \tau w \in D\left(\alpha \eta, r^{\alpha}\right)$. Thus (4.3) gives

$$
E_{\alpha}(\tau w)=\frac{1}{2 \pi i} \int_{\gamma(\eta, r)} \frac{\zeta^{\alpha-1} e^{\zeta}}{\zeta^{\alpha}-\tau w} d \zeta .
$$

Since $\overline{E_{\alpha}(z)}=E_{\alpha}(\bar{z})$ for all $z$, we obtain

$$
\begin{gathered}
-2 \pi^{2} \Phi_{K}(y)=\int_{0}^{\infty} \frac{1}{\operatorname{Im} w} \operatorname{Im}\left(\frac{E_{\alpha}(\tau w)}{w}\right) d u \\
=-\frac{1}{2 \pi i} \int_{0}^{\infty}\left\{\frac{1}{|w|^{2}} \int_{\gamma(\eta, r)} \frac{\left(\zeta^{\alpha}-2 \tau \operatorname{Re} w\right) \zeta^{\alpha-1} e^{\zeta}}{\left(\zeta^{\alpha}-\tau w\right)\left(\zeta^{\alpha}-\tau \bar{w}\right)} d \zeta\right\} d u .
\end{gathered}
$$


Let $0<t<1$. A change of variable $u \longrightarrow t u$ yields

$$
-2 \pi^{2} \Phi_{K}(t y)=-\frac{1}{2 \pi i t} \int_{0}^{\infty}\left\{\frac{1}{|y|^{2}+u^{2}} \int_{\gamma(\eta, r)} \frac{\left(\zeta^{\alpha}-2 \tau t y_{3}\right) \zeta^{\alpha-1} e^{\zeta}}{\left(\zeta^{\alpha}-\tau t w\right)\left(\zeta^{\alpha}-\tau t \bar{w}\right)} d \zeta\right\} d u
$$

Divide $\gamma(\eta, r)$ as $\gamma_{1}+\gamma_{2}+\gamma_{3}$ where

$$
\begin{gathered}
\gamma_{1}: \zeta=s e^{i \eta}, r \leq s<\infty \\
-\gamma_{2}: \zeta=s e^{-i \eta}, r \leq s<\infty \\
\gamma_{3}: \zeta=r e^{i \varphi},|\varphi| \leq \eta
\end{gathered}
$$

Then from (5.2) and (5.3) we have the expression

$$
4 \pi^{3} i R\left(y ; \alpha_{n}, \tau_{n}\right) \equiv R_{1}+R_{2}+R_{3}
$$

where

$$
R_{j}=\int_{0}^{1}\left(\int_{0}^{\infty}\left\{\frac{1}{|y|^{2}+u^{2}} \int_{\gamma_{j}} \frac{\left(\zeta^{\alpha}-2 \tau t y_{3}\right) \zeta^{\alpha-1} e^{\zeta}}{\left(\zeta^{\alpha}-\tau t w\right)\left(\zeta^{\alpha}-\tau t \bar{w}\right)} d \zeta\right\} d u \frac{J_{1}(\lambda|y| \sqrt{1-t})}{\sqrt{t(1-t)}}\right) d t
$$

From a simple geometrical observation, for all $t \in[0,1]$ and $u \geq 0$ we have the estimates $\left|e^{i \alpha \eta}-t w\right| \geq \sin (\theta-\alpha \eta)$ and $\left|e^{i \alpha \eta}-t \bar{w}\right| \geq \sin (\theta-\alpha \eta)$. Note that the common right-hand side of theses estimates gives the distance between the point $e^{i \alpha \eta}$ and the set $\{w \mid \theta \leq$ $|\arg w| \leq \pi\}$.

Then these estimates together with a change of variable yield

$$
\begin{gathered}
\left|\int_{\gamma_{1}} \frac{\left(\zeta^{\alpha}-2 \tau t y_{3}\right) \zeta^{\alpha-1} e^{\zeta}}{\left(\zeta^{\alpha}-\tau t w\right)\left(\zeta^{\alpha}-\tau t \bar{w}\right)} d \zeta\right| \leq \int_{r}^{\infty} \frac{s^{\alpha-1} e^{s \cos \eta}\left(s^{\alpha}+2 \tau\left|y_{3}\right|\right) d s}{\left|s^{\alpha} e^{i \alpha \eta}-\tau t w\right|\left|s^{\alpha} e^{i \alpha \eta}-\tau t \bar{w}\right|} \\
=\frac{1}{\alpha} \int_{r^{\alpha} / \tau}^{\infty} \frac{e^{\tau^{1 / \alpha} \xi^{1 / \alpha} \cos \eta}\left(\xi+2\left|y_{3}\right|\right) d \xi}{\left|e^{i \alpha \eta}-t w\right|\left|e^{i \alpha \eta}-t \bar{w}\right|} \\
\leq \frac{1}{\alpha \sin ^{2}(\theta-\alpha \eta)} \int_{0}^{\infty} e^{\tau^{1 / \alpha} \xi^{1 / \alpha} \cos \eta}\left(\xi+2\left|y_{3}\right|\right) d \xi \\
\leq \frac{1}{\alpha \sin ^{2}(\theta-\alpha \eta) \tau^{1 / \alpha}} \int_{0}^{\infty} e^{s \cos \eta}\left(\tau^{-1 / \alpha} s+2 R^{\alpha}\right) d s=O\left(\tau^{-1 / \alpha}\right) .
\end{gathered}
$$

Similarly we have the same estimate for the integral of the same function on $\gamma_{2}$. Therefore, using the well known estimate

$$
\left|J_{1}(s)\right| \leq \frac{s}{2}, s \geq 0
$$

we obtain, for $j=1,2 R_{j}=O\left(\tau^{-1 / \alpha}\right)$ as $\tau \longrightarrow \infty$. This is a uniform estimate with $y$ satisfying (4.9) and (4.10) for $x=0$ and $\omega=(0,0,1)^{T}$.

Next we show that $R_{3}=O\left(\tau^{-1 / 2}\right)$ as $\tau \longrightarrow \infty$. 
We have

$$
\begin{gathered}
I(w, \tau t) \equiv\left|\int_{\gamma_{3}} \frac{\left(\zeta^{\alpha}-2 \tau t y_{3}\right) \zeta^{\alpha-1} e^{\zeta}}{\left(\zeta^{\alpha}-\tau t w\right)\left(\zeta^{\alpha}-\tau t \bar{w}\right)} d \zeta\right| \\
\leq r^{\alpha} \int_{-\eta}^{\eta} e^{r \cos \varphi} \frac{\left|r^{\alpha} e^{i \alpha \varphi}-2 \tau t y_{3}\right| d \varphi}{\left|r^{\alpha} e^{i \alpha \varphi}-\tau t w\right|\left|r^{\alpha} e^{i \alpha \varphi}-\tau t \bar{w}\right|} \\
\leq(r R)^{\alpha}(1+2 \tau t) \int_{-\eta}^{\eta} \frac{d \varphi}{\left|r^{\alpha} e^{i \alpha \varphi}-\tau t w\right|\left|r^{\alpha} e^{i \alpha \varphi}-\tau t \bar{w}\right|}
\end{gathered}
$$

and thus this yields

$$
\begin{gathered}
\left|R_{3}\right| \leq \int_{0}^{1}\left(\int_{0}^{\infty} \frac{I(w, \tau t) d u}{|y|^{2}+u^{2}}\right) \frac{\left|J_{1}(\lambda|y| \sqrt{1-t})\right| d t}{\sqrt{t(1-t)}} \\
\leq(r R)^{\alpha} \frac{\lambda R^{\alpha}}{2} \int_{0}^{1} \frac{1+2 \tau t}{\sqrt{t}} d t \int_{0}^{\infty} \frac{d u}{|y|^{2}+u^{2}}\left(\int_{-\eta}^{\eta} \frac{d \varphi}{\left|r^{\alpha} e^{i \alpha \varphi}-\tau t w\right|\left|r^{\alpha} e^{i \alpha \varphi}-\tau t \bar{w}\right|}\right) .
\end{gathered}
$$

A change of variable gives

$$
\begin{gathered}
\int_{0}^{1} \frac{1+2 \tau t}{\sqrt{t}} d t \int_{0}^{\infty} \frac{d u}{|y|^{2}+u^{2}}\left(\int_{-\eta}^{\eta} \frac{d \varphi}{\left.\mid r^{\alpha} e^{i \alpha \varphi-\tau t w|| r^{\alpha} e^{i \alpha \varphi}-\tau t \bar{w} \mid}\right)}\right. \\
=\frac{1}{\sqrt{\tau}} \int_{0}^{\tau} \frac{1+2 \xi}{\sqrt{\xi}} d \xi \int_{0}^{\infty} \frac{d u}{|y|^{2}+u^{2}}\left(\int_{-\eta}^{\eta} \frac{d \varphi}{\left|r^{\alpha} e^{i \alpha \varphi}-\xi w\right|\left|r^{\alpha} e^{i \alpha \varphi}-\xi \bar{w}\right|}\right) \\
\leq \frac{1}{\sqrt{\tau}} \int_{0}^{\infty} \frac{1+2 \xi}{\sqrt{\xi}} d \xi \int_{0}^{\infty} \frac{d u}{|y|^{2}+u^{2}}\left(\int_{-\eta}^{\eta} \frac{d \varphi}{\left|r^{\alpha} e^{i \alpha \varphi}-\xi w\right|\left|r^{\alpha} e^{i \alpha \varphi}-\xi \bar{w}\right|}\right)=\frac{1}{\sqrt{\tau}}\left(I_{1}+I_{2}\right)
\end{gathered}
$$

where

$$
I_{1}=\int_{2}^{\infty} \frac{1+2 \xi}{\sqrt{\xi}} d \xi \int_{0}^{\infty} \frac{d u}{|y|^{2}+u^{2}}\left(\int_{-\eta}^{\eta} \frac{d \varphi}{\left|r^{\alpha} e^{i \alpha \varphi}-\xi w\right|\left|r^{\alpha} e^{i \alpha \varphi}-\xi \bar{w}\right|}\right)
$$

and

$$
I_{2}=\int_{0}^{2} \frac{1+2 \xi}{\sqrt{\xi}} d \xi \int_{0}^{\infty} \frac{d u}{|y|^{2}+u^{2}}\left(\int_{-\eta}^{\eta} \frac{d \varphi}{\left|r^{\alpha} e^{i \alpha \varphi}-\xi w\right|\left|r^{\alpha} e^{i \alpha \varphi}-\xi \bar{w}\right|}\right) .
$$

If $\xi \geq 2$, then we have

$$
\left|r^{\alpha} e^{i \alpha \varphi}-\xi w\right| \geq \xi|w|-r^{\alpha} \geq \xi|w|\left(1-\frac{r^{\alpha}}{\xi|w|}\right) \geq \xi|w|\left(1-\frac{r^{\alpha}}{2|y|}\right) \geq \frac{\xi|w|}{2}
$$

Therefore we get

$$
I_{1} \leq 4 \int_{2}^{\infty} \frac{1+2 \xi}{\xi^{2+1 / 2}} d \xi \int_{0}^{\infty} \frac{d u}{\left(r^{2 \alpha}+u^{2}\right)^{2}}<\infty .
$$

On the other hand, a geometrical observation gives, for all $\xi>0$

$$
\left|r^{\alpha} e^{i \alpha \varphi}-\xi w\right| \geq r^{\alpha} \sin (\theta-\alpha \eta)
$$


This right-hand side is just the distance between two sets $\{w|\theta \leq| \arg w \mid \leq \pi\}$ and $\gamma_{3}$ in the complex plane. Therefore we obtain

$$
I_{2} \leq \frac{2 \eta}{r^{2 \alpha} \sin ^{2}(\theta-\alpha \eta)} \int_{0}^{2} \frac{1+2 \xi}{\sqrt{\xi}} d \xi \int_{0}^{\infty} \frac{d u}{r^{2 \alpha}+u^{2}}<\infty .
$$

Now from (5.5) to (5.8) we obtain the desired estimate for $R_{3}$.

Summing up, we have $R=O\left(\tau^{-1 / 2}\right)$ as $\tau \longrightarrow \infty$ and this is a uniform estimate with $y$ satisfying (4.9) and (4.10) for $x=0$ and $\omega=(0,0,1)^{T}$. Therefore the function $v^{\lambda}\left(\cdot ; \alpha, \tau, \vartheta_{1}, \vartheta_{2}\right)$ converges to the function $v(y)$ uniformly for $y$ in any compact subset of $\mathbf{R}^{3} \backslash \overline{C_{0}(\pi \alpha / 2, \omega)}$. Since $\alpha$ can be arbitrary small and the function $v^{\lambda}\left(\cdot ; \alpha, \tau, \vartheta_{1}, \vartheta_{2}\right)$ satisfies the elliptic equation $\triangle u+\lambda^{2} u=0$, a standard argument yields that, for suitably chosen sequences $\left\{\alpha_{n}\right\}$ and $\left\{\tau_{n}\right\}$ the sequence $v^{\lambda}\left(\cdot ; \alpha_{n}, \tau_{n}, \vartheta_{1}, \vartheta_{2}\right)$ converges to $v(y)$ in $H_{\mathrm{loc}}^{1}(\Omega \backslash \sigma)$.

The next point of the proof is that the right-hand side of (5.1) coincides with $G_{\lambda}(y)$. This is a direct computation. The second term of the right hand side of (5.1) becomes

$$
\frac{\lambda}{8 \pi} \int_{0}^{1} J_{1}(\lambda|y-x| \sqrt{1-t}) \frac{d t}{\sqrt{t(1-t)}}=\frac{\lambda}{4 \pi} \int_{0}^{1}\left(1-w^{2}\right)^{-1 / 2} J_{1}(\lambda|y-x| w) d w .
$$

Here we know that

$$
s \int_{0}^{1}\left(1-w^{2}\right)^{-1 / 2} J_{1}(s w) d w=1-\cos s .
$$

This is an implication of (30) for $\beta=0, a=s$ and $\nu=1$ on p.337 of [5]:

$$
\int_{0}^{s}\left(s^{2}-x^{2}\right)^{-1 / 2} J_{1}(x) d x=\frac{\pi}{2}\left\{J_{1 / 2}\left(\frac{s}{2}\right)\right\}^{2}
$$

where $J_{1 / 2}$ stands for the Bessel function of order $1 / 2$ and has the explicit form

$$
J_{1 / 2}(w)=\sqrt{\frac{2}{\pi w}} \sin w, w>0 .
$$

Using (5.1), (5.9) and (5.10), we conclude that $v(y)=G_{\lambda}(y)$.

Definition 5.3. We call the needle sequence $\left\{\left.v^{\lambda}\left(\cdot-x ; \alpha_{n}, \tau_{n}, \vartheta_{1}, \vartheta_{2}\right)\right|_{\Omega}\right\}$ given in Theorem 5.1 a standard needle sequence for $(x, \sigma)$ for the Helmholtz equation $\triangle u+\lambda^{2} u=0$.

Therefore, now we can say that the probe method applied to the inverse obstacle scattering problems becomes a completely explicit method if one uses only straight needles and standard needle sequences. In this case this explicit method gives information on the location and shape of unknown obstacles more than the convex hull.

Some remarks are in order.

Remark 5.2. From Theorem 4.3 and (5.10) we get the precise values of $v^{\lambda}\left(y-x ; \alpha, \tau, \vartheta_{1}, \vartheta_{2}\right)$ on the line $y=x+s \omega(-\infty<s<\infty)$ with $\omega=\vartheta_{1} \times \vartheta_{2}$. The results are:

if $y=x+s \omega$ with $s \neq 0$, then

$$
\begin{gathered}
v^{\lambda}\left(y-x ; \alpha, \tau, \vartheta_{1}, \vartheta_{2}\right)=\frac{1}{4 \pi} \frac{E_{\alpha}(\tau s)-\cos \lambda s}{s} \\
-\frac{\lambda}{4 \pi} \int_{0}^{1}\left(1-w^{2}\right)^{-1 / 2} E_{\alpha}\left(\tau\left(1-w^{2}\right) s\right) J_{1}(\lambda s w) d w
\end{gathered}
$$


if $y=x$, then

$$
\left.v^{\lambda}\left(y-x ; \alpha, \tau, \vartheta_{1}, \vartheta_{2}\right)\right|_{y=x}=\frac{\tau}{4 \pi \Gamma(1+\alpha)} .
$$

Moreover, from (4.16) and the fact that $J_{1}(s)$ is an odd function we see that $\nabla v^{\lambda}(y-$ $\left.x ; \alpha, \tau, \vartheta_{1}, \vartheta_{2}\right)$ on the line $y=x+s \omega(-\infty<s<\infty)$ is also parallel to $\omega$. In particular, we have

$$
\left.\nabla v^{\lambda}\left(y-x ; \alpha, \tau, \vartheta_{1}, \vartheta_{2}\right)\right|_{y=x}=\frac{\tau^{2}}{4 \pi \Gamma(1+2 \alpha)} \omega
$$

Remark 5.3. Since the function

$$
i \frac{\sin \lambda|y|}{4 \pi|y|}
$$

satisfies the Helmholtz equation in the whole space, we see that the sequence $\left\{\tilde{v}^{\lambda}(y-\right.$ $\left.\left.x ; \alpha_{n}, \tau_{n}, \vartheta_{1}, \vartheta_{2}\right)\right\}$ given by

$$
v^{\lambda}\left(y-x ; \alpha_{n}, \tau_{n}, \vartheta_{1}, \vartheta_{2}\right)+i \frac{\sin \lambda|y-x|}{4 \pi|y-x|}, n=1, \cdots, y \in \Omega
$$

is also a needle sequence for $(x, \sigma)$ with $G=\tilde{G}_{\lambda}$ given by

$$
\tilde{G}_{\lambda}(y)=\frac{e^{i \lambda|y|}}{4 \pi|y|}
$$

This $\tilde{G}_{\lambda}$ is nothing but the fundamental solution for the Helmholtz equation and satisfies (2.1) for any finite cone $V$ with vertex at 0 . We also call the sequence $\left\{\tilde{v}^{\lambda}(y-\right.$ $\left.\left.x ; \alpha_{n}, \tau_{n}, \vartheta_{1}, \vartheta_{2}\right)\right\}$ the standard needle sequence for $(x, \sigma)$.

Remark 5.4. Fix $\alpha \in] 0,1[$. As a corollary of the proof of Theorem 5.1 we see that the function

$$
\Phi(y)=G_{\lambda}(y)-v^{\lambda}\left(y ; \alpha, \tau, \vartheta_{1}, \vartheta_{2}\right), y \neq 0
$$

with a large parameter $\tau$ is a Carleman function (for the Helmholtz equation) for a special domain $\Omega$ whose boundary consists of a part of the conic surface and a smooth surface $\Gamma$ lying inside the cone of axis direction $\vartheta_{1} \times \vartheta_{2}$ and aperture angle $\pi \alpha$. See Figure 2 for an illustration of $\Omega$. This is an extension of a result in [36] to the Helmholtz equation and new. 


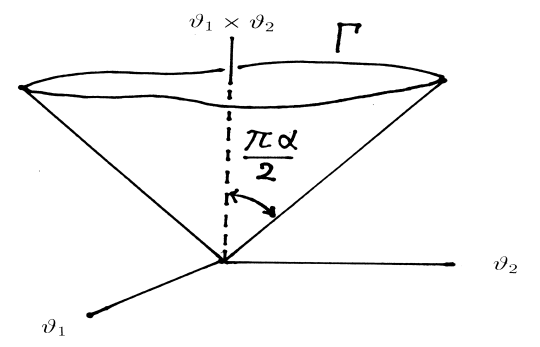

Figure 2: An illustration of $\Omega$.

As pointed out in the beginning of this section Theorem 4.1 for $\lambda>0$ does not give this result.

Remark 5.5. For a recent application of the Vekua transform to a boundary value problem for the Helmholtz equation see [9]. In [22], we applied the idea of the Vekua transform to the construction of the Herglotz wave function that approximates in a disc a complex exponential solution of the Helmholtz equation. This is an application to inverse obstacle scattering problems in two dimensions.

\section{Conclusion and open problems}

We could find an explicit needle sequence for a needle given by a segment in three dimensions. This makes the probe method completely explicit as same as the enclosure method. It is based on a principle that connects the regular part of a Carleman function with a needle sequence in the probe method.

Some further problems to be solved are in order.

- A mathematically interesting question is: can one give an explicit needle sequence having a closed-form for a general needle not necessary given by a segment in three dimensions? - Using the standard needle sequence for the Helmholtz equation $\Delta u+\lambda^{2} u=0$, one can construct the corresponding indicator sequence for some inverse boundary value problems that are the reduction of inverse obstacle scattering problems at a fixed wave number to a bounded domain. Can one prove the blowing up of the indicator sequence without 
assuming that $\lambda$ is small when the tip of the needle is located on the boundary, inside or passing through unknown obstacles. For the case when $\lambda$ is small see $[21,24]$.

- Construct a needle sequence for the stationary Schrödinger equation $-\Delta u+V(x) u=0$ or the equation $\nabla \cdot \gamma(x) \nabla u=0$ as explicit as possible. This is important for the reconstruction problem of discontinuity embedded in an inhomogeneous conductive medium. See [19] for the probe method applied to this problem.

- Do the numerical implementation of the probe method by using the standard needle sequences and compare the results with the previous numerical study done in $[10,12]$ of the probe method in two dimensions.

\section{Acknowledgement}

This research was partially supported by Grant-in-Aid for Scientific Research (C)(No. 18540160) of Japan Society for the Promotion of Science. The author wishes to thank the anonymous referees for their comments and suggestions.

\section{A Appendix. Proof of Theorem 4.1}

\section{A.1 Preliminaries}

Given an entire function $K(w)$ set

$$
\Phi(x)=\int_{0}^{\infty} \frac{K(w)}{w} \frac{\Psi(\lambda u)}{\sqrt{\left|x^{\prime}\right|^{2}+u^{2}}} d u
$$

where $\Psi$ is a smooth function on $[0, \infty[$ and

$$
w=x_{3}+i \sqrt{\left|x^{\prime}\right|^{2}+u^{2}} .
$$

Set

$$
f(w)=\frac{K(w)}{w} .
$$

Here we show that under suitable growth conditions for $f(w)$ as $u \longrightarrow \infty$ and the choice of $\Psi(\lambda u)$ the function $\Phi(x)$ satisfies

$$
\triangle \Phi+\lambda^{2} \Phi=0
$$

for $x^{\prime} \neq 0$.

First we do the following formal computations. We have

$$
\frac{\partial^{2} \Phi}{\partial x_{3}^{2}}=\int_{0}^{\infty} f^{\prime \prime}(w) \frac{\Psi(\lambda u)}{\sqrt{\left|x^{\prime}\right|^{2}+u^{2}}} d u
$$

Let $k=1,2$. We have

$$
\frac{\partial \Phi}{\partial x_{k}}=\int_{0}^{\infty} f^{\prime}(w) i \frac{x_{k}}{\left|x^{\prime}\right|^{2}+u^{2}} \Psi(\lambda u) d u-\int_{0}^{\infty} f(w) \frac{x_{k}}{\left(\sqrt{\left|x^{\prime}\right|^{2}+u^{2}}\right)^{3}} \Psi(\lambda u) d u
$$


Then we obtain

$$
\begin{aligned}
& \frac{\partial^{2} \Phi}{\partial x_{k}^{2}}=-\int_{0}^{\infty} f^{\prime \prime}(w) \frac{x_{k}^{2}}{\left(\sqrt{\left|x^{\prime}\right|^{2}+u^{2}}\right)^{3}} \Psi(\lambda u) d u+\int_{0}^{\infty} f^{\prime}(w) i \frac{\Psi(\lambda u) d u}{\left|x^{\prime}\right|^{2}+u^{2}} \\
& -\int_{0}^{\infty} f^{\prime}(w) i \frac{2 x_{k}^{2}}{\left(\left|x^{\prime}\right|^{2}+u^{2}\right)^{2}} \Psi(\lambda u) d u-\int_{0}^{\infty} f^{\prime}(w) i \frac{x_{k}^{2}}{\left(\left|x^{\prime}\right|^{2}+u^{2}\right)^{2}} \Psi(\lambda u) d u \\
& -\int_{0}^{\infty} f(w) \frac{\Psi(\lambda u) d u}{\left(\sqrt{\left|x^{\prime}\right|^{2}+u^{2}}\right)^{3}}+\int_{0}^{\infty} f(w) 3 \frac{x_{k}^{2}}{\left(\sqrt{\left|x^{\prime}\right|^{2}+u^{2}}\right)^{5}} \Psi(\lambda u) d u .
\end{aligned}
$$

This yields

$$
\begin{aligned}
& \sum_{k=1}^{2} \frac{\partial^{2} \Phi}{\partial x_{k}^{2}}=-\int_{0}^{\infty} f^{\prime \prime}(w) \frac{\left|x^{\prime}\right|^{2}}{\left(\sqrt{\left|x^{\prime}\right|^{2}+u^{2}}\right)^{3}} \Psi(\lambda u) d u+2 i \int_{0}^{\infty} f^{\prime}(w) \frac{\Psi(\lambda u) d u}{\left|x^{\prime}\right|^{2}+u^{2}} \\
& -2 i \int_{0}^{\infty} f^{\prime}(w) \frac{\left|x^{\prime}\right|^{2}}{\left(\left|x^{\prime}\right|^{2}+u^{2}\right)^{2}} \Psi(\lambda u) d u-i \int_{0}^{\infty} f^{\prime}(w) \frac{\left|x^{\prime}\right|^{2}}{\left(\left|x^{\prime}\right|^{2}+u^{2}\right)^{2}} \Psi(\lambda u) d u \\
& -2 \int_{0}^{\infty} f(w) \frac{\Psi(\lambda u) d u}{\left(\sqrt{\left|x^{\prime}\right|^{2}+u^{2}}\right)^{3}}+3 \int_{0}^{\infty} f(w) \frac{\left|x^{\prime}\right|^{2}}{\left(\sqrt{\left.\left|x^{\prime}\right|^{2}+u^{2}\right)^{5}} \Psi(\lambda u) d u\right.} \\
& =\int_{0}^{\infty} f(w)\left\{\frac{3\left|x^{\prime}\right|^{2}}{\left(\sqrt{\left|x^{\prime}\right|^{2}+u^{2}}\right)^{5}}-\frac{2}{\left(\sqrt{\left|x^{\prime}\right|^{2}+u^{2}}\right)^{3}}\right\} \Psi(\lambda u) d u \\
& +i \int_{0}^{\infty} f^{\prime}(w)\left\{\frac{2}{\left|x^{\prime}\right|^{2}+u^{2}}-\frac{3\left|x^{\prime}\right|^{2}}{\left(\left|x^{\prime}\right|^{2}+u^{2}\right)^{2}}\right\} \Psi(\lambda u) d u \\
& -\int_{0}^{\infty} f^{\prime \prime}(w) \frac{\left|x^{\prime}\right|^{2}}{\left(\sqrt{\left|x^{\prime}\right|^{2}+u^{2}}\right)^{3}} \Psi(\lambda u) d u \\
& -\int_{0}^{\infty} f^{\prime \prime}(w) \frac{\left|x^{\prime}\right|^{2}}{\left(\sqrt{\left|x^{\prime}\right|^{2}+u^{2}}\right)^{3}} \Psi(\lambda u) d u . \\
& =\int_{0}^{\infty} f(w) \frac{\left|x^{\prime}\right|^{2}-2 u^{2}}{\left(\sqrt{\left|x^{\prime}\right|^{2}+u^{2}}\right)^{5}} \Psi(\lambda u) d u+i \int_{0}^{\infty} f^{\prime}(w) \frac{2 u^{2}-\left|x^{\prime}\right|^{2}}{\left(\left|x^{\prime}\right|^{2}+u^{2}\right)^{2}} \Psi(\lambda u) d u
\end{aligned}
$$


From (A.1) and (A.2) we have

$$
\begin{aligned}
& \triangle \Phi=\int_{0}^{\infty} f(w) \frac{\left|x^{\prime}\right|^{2}-2 u^{2}}{\left(\sqrt{\left|x^{\prime}\right|^{2}+u^{2}}\right)^{5}} \Psi(\lambda u) d u+i \int_{0}^{\infty} f^{\prime}(w) \frac{2 u^{2}-\left|x^{\prime}\right|^{2}}{\left(\left|x^{\prime}\right|^{2}+u^{2}\right)^{2}} \Psi(\lambda u) d u \\
& +\int_{0}^{\infty} f^{\prime \prime}(w)\left\{\frac{1}{\sqrt{\left|x^{\prime}\right|^{2}+u^{2}}}-\frac{\left|x^{\prime}\right|^{2}}{\left(\sqrt{\left|x^{\prime}\right|^{2}+u^{2}}\right)^{3}}\right\} \Psi(\lambda u) d u \\
& =\int_{0}^{\infty} f(w) \frac{\left|x^{\prime}\right|^{2}-2 u^{2}}{\left(\sqrt{\left|x^{\prime}\right|^{2}+u^{2}}\right)^{5}} \Psi(\lambda u) d u+i \int_{0}^{\infty} f^{\prime}(w) \frac{2 u^{2}-\left|x^{\prime}\right|^{2}}{\left(\left|x^{\prime}\right|^{2}+u^{2}\right)^{2}} \Psi(\lambda u) d u \\
& +\int_{0}^{\infty} f^{\prime \prime}(w) \frac{u^{2}}{\left(\sqrt{\left|x^{\prime}\right|^{2}+u^{2}}\right)^{3}} \Psi(\lambda u) d u .
\end{aligned}
$$

Integration by parts yields

$$
\begin{aligned}
& \int_{0}^{\infty} f^{\prime \prime}(w) \frac{u^{2}}{\left(\sqrt{\left|x^{\prime}\right|^{2}+u^{2}}\right)^{3}} \Psi(\lambda u) d u=-i \int_{0}^{\infty} \frac{\partial}{\partial u}\left\{f^{\prime}(w)\right\} \frac{u}{\left|x^{\prime}\right|^{2}+u^{2}} \Psi(\lambda u) d u \\
& =-\left.i f^{\prime}(w) \frac{u}{\left|x^{\prime}\right|^{2}+u^{2}} \Psi(\lambda u)\right|_{0} ^{\infty}+i \int_{0}^{\infty} f^{\prime}(w) \frac{\partial}{\partial u}\left\{\frac{u \Psi(\lambda u)}{\left|x^{\prime}\right|^{2}+u^{2}}\right\} d u \\
& =i \int_{0}^{\infty} f^{\prime}(w) \frac{\left|x^{\prime}\right|^{2}-u^{2}}{\left(\left|x^{\prime}\right|^{2}+u^{2}\right)^{2}} \Psi(\lambda u) d u+i \lambda \int_{0}^{\infty} f^{\prime}(w) \frac{u \Psi^{\prime}(\lambda u)}{\left|x^{\prime}\right|^{2}+u^{2}} d u .
\end{aligned}
$$

Note that we assumed that

$$
\lim _{u \longrightarrow \infty} f^{\prime}(w) \frac{u}{\left|x^{\prime}\right|^{2}+u^{2}} \Psi(\lambda u)=0 .
$$

In what follows we always assume this type of growth condition for $f(w)$ to delete a term coming from integration by parts.

Therefore (A.3) becomes

$$
\begin{aligned}
& \triangle \Phi=\int_{0}^{\infty} f(w) \frac{\left|x^{\prime}\right|^{2}-2 u^{2}}{\left(\sqrt{\left|x^{\prime}\right|^{2}+u^{2}}\right)^{5}} \Psi(\lambda u) d u \\
& +i \int_{0}^{\infty} f^{\prime}(w) \frac{u^{2} \Psi(\lambda u)}{\left(\left|x^{\prime}\right|^{2}+u^{2}\right)^{2}} d u+i \lambda \int_{0}^{\infty} f^{\prime}(w) \frac{u \Psi^{\prime}(\lambda u)}{\left|x^{\prime}\right|^{2}+u^{2}} d u .
\end{aligned}
$$

Integration by parts yields

$$
\begin{aligned}
& i \int_{0}^{\infty} f^{\prime}(w) \frac{u^{2} \Psi(\lambda u)}{\left(\left|x^{\prime}\right|^{2}+u^{2}\right)^{2}}=\int_{0}^{\infty} \frac{\partial}{\partial u}\{f(w)\} \frac{u \Psi(\lambda u)}{\left(\sqrt{\left|x^{\prime}\right|^{2}+u^{2}}\right)^{3}} d u \\
& =\left.f(w) \frac{u \Psi(\lambda u)}{\left(\sqrt{\left|x^{\prime}\right|^{2}+u^{2}}\right)^{3}}\right|_{0} ^{\infty}-\int_{0}^{\infty} f(w) \frac{\partial}{\partial u}\left\{\frac{u \Psi(\lambda u)}{\left(\sqrt{\left|x^{\prime}\right|^{2}+u^{2}}\right)^{3}}\right\} d u \\
& =-\int_{0}^{\infty} f(w) \frac{\left|x^{\prime}\right|^{2}-2 u^{2}}{\left(\sqrt{\left|x^{\prime}\right|^{2}+u^{2}}\right)^{5}} \Psi(\lambda u) d u-\lambda \int_{0}^{\infty} f(w) \frac{u \Psi^{\prime}(\lambda u)}{\left(\sqrt{\left|x^{\prime}\right|^{2}+u^{2}}\right)^{3}} d u .
\end{aligned}
$$


Therefore we obtain

$$
\triangle \Phi=-\lambda \int_{0}^{\infty} f(w) \frac{u \Psi^{\prime}(\lambda u)}{\left(\sqrt{\left|x^{\prime}\right|^{2}+u^{2}}\right)^{3}} d u+i \lambda \int_{0}^{\infty} f^{\prime}(w) \frac{u \Psi^{\prime}(\lambda u)}{\left|x^{\prime}\right|^{2}+u^{2}} d u .
$$

Here we assume that

$$
\Psi^{\prime}(0)=0
$$

Then integration by parts yields

$$
\begin{aligned}
& i \int_{0}^{\infty} f^{\prime}(w) \frac{u \Psi^{\prime}(\lambda u)}{\left|x^{\prime}\right|^{2}+u^{2}} d u=\int_{0}^{\infty} \frac{\partial}{\partial u}\{f(w)\} \frac{\Psi^{\prime}(\lambda u)}{\sqrt{\left|x^{\prime}\right|^{2}+u^{2}}} d u \\
& =\left.f(w) \frac{\Psi^{\prime}(\lambda u)}{\sqrt{\left|x^{\prime}\right|^{2}+u^{2}}}\right|_{0} ^{\infty}-\int_{0}^{\infty} f(w) \frac{\partial}{\partial u} \frac{\Psi^{\prime}(\lambda u)}{\sqrt{\left|x^{\prime}\right|^{2}+u^{2}}} d u \\
& =\int_{0}^{\infty} f(w) \frac{u \Psi^{\prime}(\lambda u)}{\left(\sqrt{\left|x^{\prime}\right|^{2}+u^{2}}\right)^{3}} d u-\lambda \int_{0}^{\infty} f(w) \frac{\Psi^{\prime \prime}(\lambda u)}{\sqrt{\left|x^{\prime}\right|^{2}+u^{2}}} d u
\end{aligned}
$$

and thus we obtain

$$
\triangle \Phi=-\lambda^{2} \int_{0}^{\infty} f(w) \frac{\Psi^{\prime \prime}(\lambda u)}{\sqrt{\left|x^{\prime}\right|^{2}+u^{2}}} d u .
$$

Therefore if $\Psi$ satisfies (A.4) and

$$
\Psi^{\prime \prime}=\Psi
$$

then from (A.5) we obtain

$$
\triangle \Phi+\lambda^{2} \Phi=0
$$

for $x^{\prime} \neq 0$.

The $\Psi$ that satisfies (A.4) and (A.6) is given by

$$
C\left(e^{u}+e^{-u}\right)
$$

and thus we choose

$$
\Psi(\lambda u)=C\left(e^{\lambda u}+e^{-\lambda u}\right)
$$

where $C$ is a constant. Then it is easy to check that the formal computations given above can be justified under the growth condition (4.1).

Remark A.1. We give some historical remarks. In [32] he considered the case when $\lambda=0$. Therein he gave a proof for the fact that $\Phi$ is harmonic for $x^{\prime} \neq 0$. However, the proof uses a change of variables and can not cover the case when $K(w)$ satisfies (4.1) with $\lambda=0$, for example, $K(w)=e^{m w}$ with $m>0$ as pointed out in Appendix A of [17]. However, in [33], he had given a direct proof in that case. Using the idea of the proof, one can easily obtain that, in the case when $\lambda=0 \Phi$ is harmonic for $x^{\prime} \neq 0$ provided

$$
\forall R>0 \sup _{|\operatorname{Re} w|<R}\left|K^{(m)}(w)\right|<\infty
$$

for $m=0,1,2$.

Except for the explanation for the choice of a suitable $\Psi$ the proof presented in this subsection follows that of [33]. 


\section{A.2 Extracting singularity at $x=0$}

We assume that $K(w)$ is real for real $w$.

Set

$$
\tilde{\Phi}(x)=\int_{0}^{\infty} \operatorname{Im}\left(\frac{K(w)}{w}\right) \frac{\Psi(\lambda u)}{\sqrt{\left|x^{\prime}\right|^{2}+u^{2}}} d u .
$$

Here we extract the singularity of this function at $x=0$.

Since

$$
K(w)=\sum_{n=0}^{\infty} \frac{K^{(n)}(0)}{n !} w^{n}
$$

we have

$$
\begin{aligned}
& \frac{K(w)}{w}=\frac{K(0)}{x_{3}+i \sqrt{\left|x^{\prime}\right|^{2}+u^{2}}}+K^{\prime}(0)++\sum_{n=2}^{\infty} \frac{K^{(n)}(0)}{n !}\left(x_{3}+i \sqrt{\left|x^{\prime}\right|^{2}+u^{2}}\right)^{n-1} \\
& =\frac{K(0)\left(x_{3}-i \sqrt{\left|x^{\prime}\right|^{2}+u^{2}}\right)}{|x|^{2}+u^{2}}+K^{\prime}(0) \\
& +\sum_{n=1}^{\infty} \frac{K^{(n+1)}(0)}{(n+1) !} \sum_{j=0}^{n} \frac{n !}{j !(n-j) !} x_{3}^{n-j} i^{j}\left(\left|x^{\prime}\right|^{2}+u^{2}\right)^{j / 2} .
\end{aligned}
$$

All coefficients $K^{(n)}(0), n=0,1, \cdots$ are real and this yields

$$
\begin{aligned}
& \operatorname{Im}\left(\frac{K(w)}{w}\right)=-\frac{\sqrt{\left|x^{\prime}\right|^{2}+u^{2}}}{|x|^{2}+u^{2}} K(0) \\
& +\sum_{n=1}^{\infty} \frac{K^{(n+1)}(0)}{(n+1) !} \sum_{j \text { is odd }}^{n} \frac{n !}{j !(n-j) !} x_{3}^{n-j}(-1)^{(j-1) / 2}\left(\left|x^{\prime}\right|^{2}+u^{2}\right)^{j / 2} .
\end{aligned}
$$

Therefore we obtain

$$
\begin{aligned}
& \int_{0}^{1} \operatorname{Im}\left(\frac{K(w)}{w}\right) \frac{\Psi(\lambda u)}{\sqrt{\left|x^{\prime}\right|^{2}+u^{2}}} d u=-K(0) \int_{0}^{1} \frac{\Psi(\lambda u)}{|x|^{2}+u^{2}} d u \\
& +\sum_{n=1}^{\infty} \frac{K^{(n+1)}(0)}{(n+1) !} \sum_{j \text { is odd }}^{n} \frac{n !}{j !(n-j) !} x_{3}^{n-j}(-1)^{(j-1) / 2} \int_{0}^{1}\left(\left|x^{\prime}\right|^{2}+u^{2}\right)^{(j-1) / 2} \Psi(\lambda u) d u \\
& \equiv-K(0) \int_{0}^{1} \frac{\Psi(\lambda u)}{|x|^{2}+u^{2}} d u
\end{aligned}
$$

modulo a $C^{\infty}$ function on the whole space. Since

$$
\int_{1}^{\infty} \operatorname{Im}\left(\frac{K(w)}{w}\right) \frac{\Psi(\lambda u)}{\sqrt{\left|x^{\prime}\right|^{2}+u^{2}}} d u
$$


is $C^{2}$ on the whole space provided $K(w)$ satisfies a suitable growth condition, we obtain that

$$
\tilde{\Phi}(x) \equiv-K(0) \int_{0}^{1} \frac{\Psi(\lambda u)}{|x|^{2}+u^{2}} d u
$$

modulo a $C^{2}$ function on the whole space.

Now we take

$$
\Psi(\lambda u)=\left(e^{\lambda u}+e^{-\lambda u}\right) / 2 .
$$

Let $0<|x|<1$. Then

$$
\begin{aligned}
& \int_{0}^{1} \frac{\Psi(\lambda u)}{|x|^{2}+u^{2}} d u=\frac{1}{2} \int_{-1}^{1} \frac{e^{\lambda u}}{|x|^{2}+u^{2}} d u \\
& =\left.\pi i \operatorname{Res}\left(\frac{e^{\lambda z}}{|x|^{2}+z^{2}}\right)\right|_{z=i|x|}-\frac{1}{2} \int_{z=e^{i \theta}, 0 \leq \theta \leq \pi} \frac{e^{\lambda z}}{|x|^{2}+z^{2}} d z \\
& =\frac{\pi}{2} \frac{e^{i \lambda|x|}}{|x|}-\frac{i}{2} \int_{0}^{\pi} \frac{e^{\lambda(\cos \theta+i \sin \theta)}}{|x|^{2}+e^{2 i \theta}} e^{i \theta} d \theta \equiv \frac{\pi}{2} \frac{e^{i \lambda|x|}}{|x|}
\end{aligned}
$$

modulo a $C^{\infty}$ function on $|x|<1$. Therefore from (A.7) one concludes that

$$
\tilde{\Phi}(x) \equiv-\frac{K(0) \pi}{2} \frac{e^{i \lambda|x|}}{|x|}
$$

modulo a $C^{2}$ function on the whole space provided $K(w)$ satisfies (4.1). In particular, if $K(0)=1$, then

$$
-\frac{1}{2 \pi^{2}} \tilde{\Phi}(x) \equiv \frac{e^{i \lambda|x|}}{4 \pi|x|}
$$

modulo a $C^{2}$ function on the whole domain.

Using the results in subsections, one obtains Theorem 4.1.

\section{References}

[1] Aizenberg, L., Carleman's formulas in complex analysis, Kluwer Academic Publ., London, 1993.

[2] Arubuzov, È. V. and Bukhgeım, A. L., The Carleman formula for the Hemholtz equation on the plane, Siberian Math. J., 47(2006), 425-432.

[3] Astala, K. and Päivärinta, L., Calderón's inverse conductivity problem in the plane, Ann. of Math., 163(2006), 265-299.

[4] Astala, K. and Päivärinta, L., A boundary integral equation for Calderón's inverse conductivity problem, Collect. Math., 2006, Vol. Extra, 127-139.

[5] Bateman, H., Higher Transcendental Functions, Volume II, Bateman Manuscript Project (A. Erdélyi, Editor), McGRAW-HILL, New York, 1954. 
[6] Bateman, H., Higher Transcendental Functions, Volume III, Bateman Manuscript Project (A. Erdélyi, Editor), McGRAW-HILL, New York, 1955.

[7] Brühl, M., Explicit characterization of inclusions in electrical impedance tomography, SIAM J. Math. Anal., 32(2001), 1327-1341.

[8] Calderón, A. P., On an inverse boundary value problem, in Seminar on Numerical Analysis and its Applications to Continuum Physics, ed. by Meyer W. H. and Raupp M. A. (Rio de janeiro: Brazilian Math. Soc.), p. 65-73, 1980.

[9] Charalambopoulos, A. and Dassios, G., On the Vekua pair in spheroidal geometry and its role in solving boundary value problems, Applicable Analysis, 81(2002), $85-113$.

[10] Cheng, J., Liu, J. J. and Nakamura, G., The numerical realization of the probe method for the inverse scattering problems from the near fields, Inverse Problems, 21(2005), 839-855.

[11] Evgrafov, M. A., Asymptotic estimates and entire functions, in Russian, Nauka, Moscow, 1979 .

[12] Erhard, K. and Potthast, R. A numerical study of the probe method, SIAM J. Scientific Computing, 28(2006), 1597-1612.

[13] Ikehata, M., Reconstruction of the shape of the inclusion by boundary measurements, Commun. in Partial Differential Equations, 23(1998), 1459-1474.

[14] Ikehata, M., Reconstruction of obstacle from boundary measurements, Wave Motion, 30(1999), 205-223.

[15] Ikehata, M., The probe method and its applications, Inverse problems and related topics, Nakamura, G., Saitoh, S., Seo, J. K. and Yamamoto, M. editors, CRC Press UK, 2000, 57-68.

[16] Ikehata, M., Reconstruction of the support function for inclusion from boundary measurements, J. Inv. Ill-Posed Problems, 8(2000), 367-378.

[17] Ikehata, M., Inverse conductivity problem in the infinite slab, Inverse Problems, $17(2001), 437-454$.

[18] Ikehata, M., Exponentially growing solutions and the Cauchy problem, Appl. Anal., 78(2001), no.1-2, 79-95.

[19] Ikehata, M., Reconstruction of inclusion from boundary measurements, J. Inv. IllPosed Problems, 10(2002), 37-65.

[20] Ikehata, M., Mittag-Leffler's function and extracting from Cauchy data, Inverse problems and spectral theory, ed. Isozaki, H., Contemp. Math., 348(2004), 41-52.

[21] Ikehata, M., A new formulation of the probe method and related problems, Inverse Problems, 21(2005), 413-426. 
[22] Ikehata, M., The Herglotz wave function, the Vekua transform and the enclosure method, Hiroshima Math. J., 35(2005), 485-506.

[23] Ikehata, M., Inverse crack problem and probe method, Cubo, 8(2006), 29-40.

[24] Ikehata, M., Two sides of probe method and obstacle with impedance boundary condition, Hokkaido Math. J., 35(2006), 659-681.

[25] Ikehata, M. and Nakamura, G., Slicing of a three-dimensional object from boundary measurements, Inverse Problems, 15(1999), 1243-1253.

[26] Ikehata, M. and Siltanen, S., Electrical impedance tomography and Mittag-Leffler's function, Inverse Problems, 20(2004), 1325-1348.

[27] Isakov, V., On uniqueness of recovery of a discontinuous conductivity coefficient, Comm. Pure. Appl. Math. 41(1988), 865-877.

[28] Kirsch, A., Characterization of the shape of a scattering obstacle using the spectral data of the far field operator, Inverse Problems, 14(1998), 1489-1512.

[29] Kress, R., Linear Integral equations, Springer, 1989.

[30] Vekua, I. N., Solutions of the Equation $\Delta u+\lambda^{2} u=0$, Soobshcheniga Akademii Nauk Gruz. SSR 3(4)(1942), 307-314.

[31] Vekua, I. N., Inversion of an Integral Transformation and Some Applications, Soobshcheniga Akademii Nauk Gruz. SSR 6(3)(1945), 177-183.

[32] Yarmukhamedov, Sh., Integral representations of harmonic functions in multidimensions, in Russian, Dokl. Akad. Nauk SSSR, 204(1972), 799-802.

[33] Yarmukhamedov, Sh., On a Cauchy problem for Laplace's equation, Mathematicheskie Zametki, 18(1975), 57-61.

[34] Yarmukhamedov, Sh., On the Cauchy problem for the Laplace equation, in Russian, Dokl. Akad. Nauk SSSR, 235(1977), 281-283.

[35] Yarmukhamedov, Sh., Integral representation of a CR-function and its holomorphic continuation, Dokl. Math. 51(1995), 253-255.

[36] Yarmukhamedov, Sh., A Carleman function and the Cauchy problem for the Laplace equation, Siberian Math. J., 45(2004), No. 3, 580-595.

e-mail address

ikehata@math.sci.gunma-u.ac.jp 